\title{
High internal phase emulsion as reaction medium for precipitating brushite crystals
}

\begin{abstract}
This present work was aimed at fabrication of brushite crystals using oil-in-water high internal phase emulsion as a reaction medium. The oil phase of more than 75 wt.\% was dispersed in the continuous aqueous phase. Due to the high oil volume fraction, the oil droplets were no longer spherical but were squeezed to take the shape of polyhedral. The morphology of the crystals was influenced by the structure of the emulsion and precursor concentration. The crystals were subjected to cytotoxicity test to ensure their compatibility with synoviocytes, which are cells that line the knee joints of rabbits. The crystals were able to sustain the cells for 5 days, which manifest their potential as osteoconductive coatings.
\end{abstract}

Keyword: E. Biomedical applications; High internal phase emulsion; Brushite 